\title{
Femtosecond thin-film laser amplifiers using chirped gratings
}

\author{
Meng Wang and Xinping Zhang ${ }^{\mathrm{a}, *}$ \\ a Institute of Information Photonics Technology, College of Applied Sciences, Beijing \\ University of Technology, Beijing 100124, P. R. China \\ *Email: zhangxinping@bjut.edu.cn
}




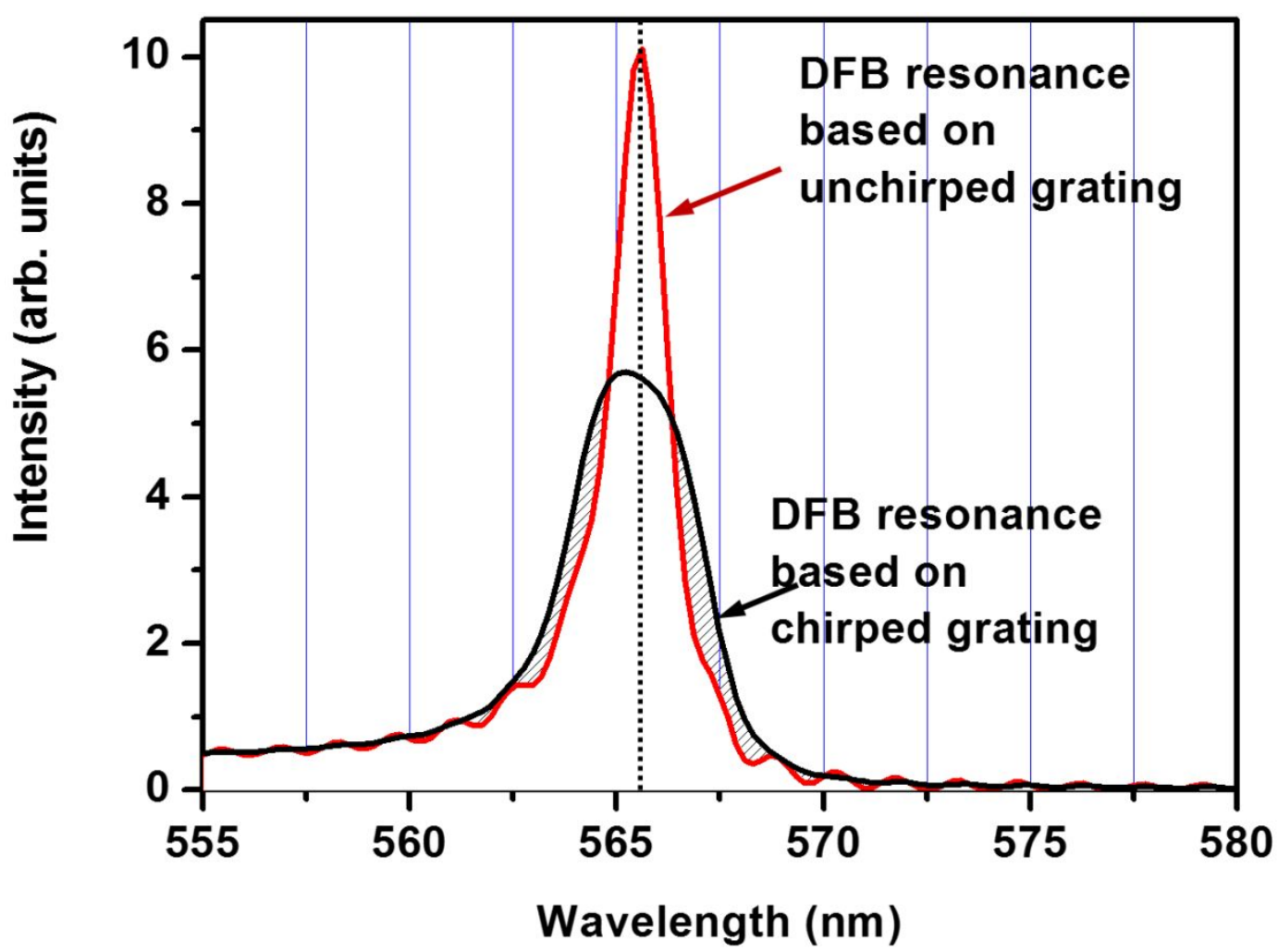

Figure S1 Calculated resonance modes of the DFB microcavities based on chirped (black) and unchirped (red) gratings. The shaded area shows the difference between the two modes due to the extension of the bandwidth for the DFB resonance mode based on chirped gratings. 


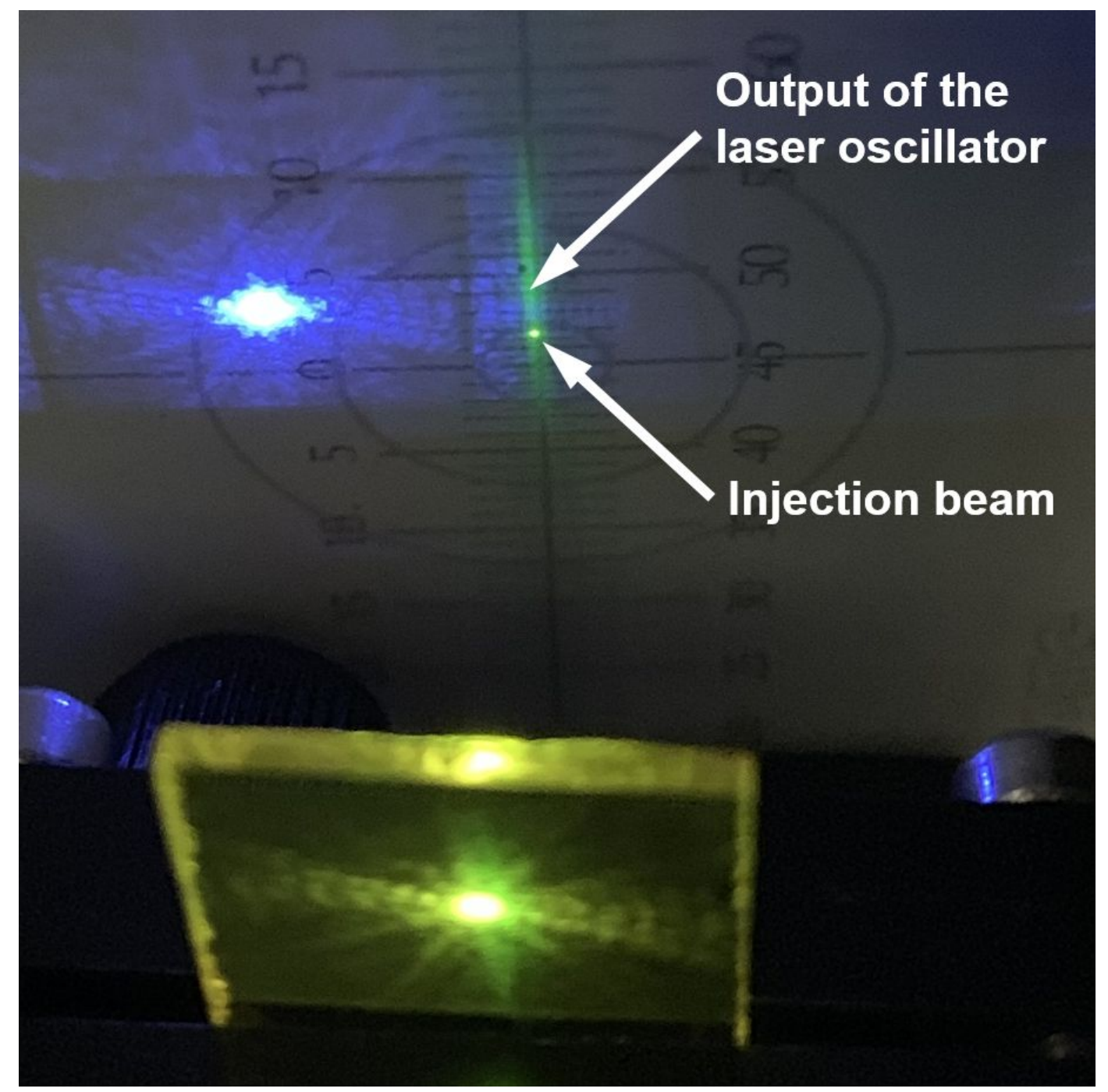

Figure S2 Photograph of the laser spots of the injection beam and the output of the laser oscillator emission. 

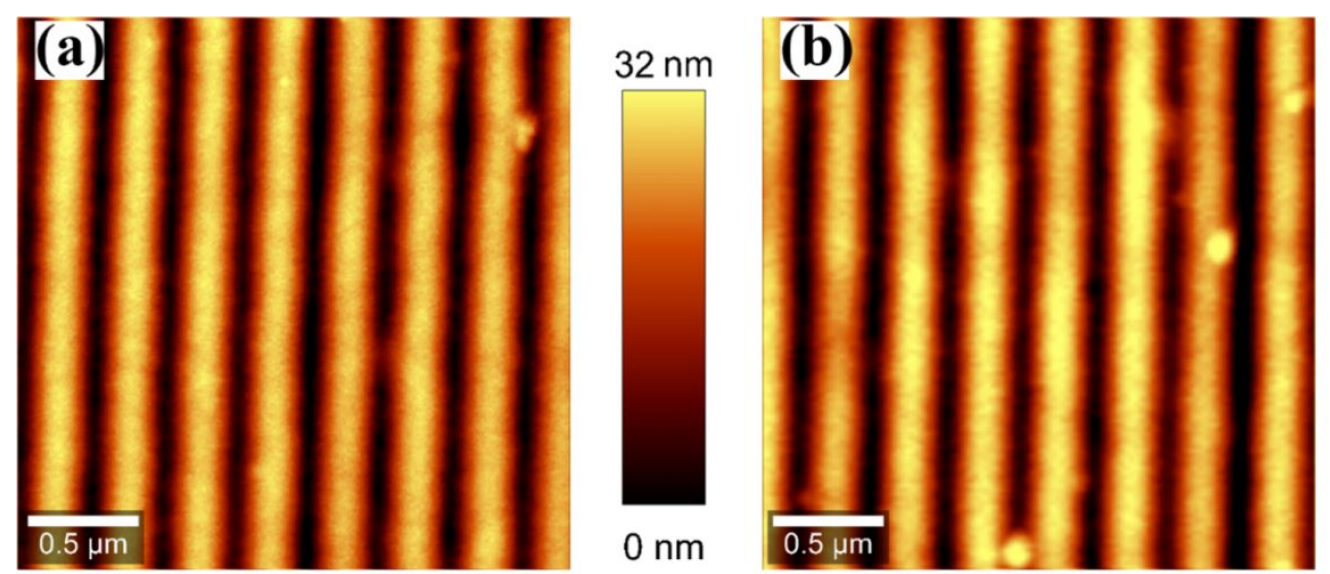

$32 \mathrm{~nm}$

Figure S3 The AFM images of the chirped gratings measured at two different locations, corresponding to a period of (a) $324 \mathrm{~nm}$ and (b) $335 \mathrm{~nm}$. 